Mathematical Research Letters 5, 577-582 (1998)

\title{
INTERSECTIONAL PAIRS OF $n$-KNOTS, LOCAL MOVES OF $n$-KNOTS, AND THEIR ASSOCIATED INVARIANTS OF $n$-KNOTS
}

\author{
EiJi Ogasa
}

\section{Introduction}

Our first purpose is to discuss the following problem. Let $S_{1}^{n+2}$ and $S_{2}^{n+2}$ be $(n+2)$-spheres embedded in the $(n+4)$-sphere $S^{n+4}(n \geqq 1)$ which intersect transversely. If we assume $M=S_{1}^{n+2} \cap S_{2}^{n+2}$ is PL homeomorphic to the single standard $n$-sphere we obtain a pair of $n$-knots, $M$ in $S_{1}^{n+2}$ and $M$ in $S_{2}^{n+2}$. We consider which pairs of $n$-knots we obtain as above. That is, let $\left(K_{1}, K_{2}\right)$ be a pair of $n$-knots. Then we consider whether the pair of $n$-knots $\left(K_{1}, K_{2}\right)$ is obtained as above. We give a complete answer to this problem (Theorem 3.1).

In order to get the complete answer, we introduce a local move of $n$-knots $(n \geq 1)$. Furthermore, we show a relation between the local move and some invariants of $n$-knots (Theorem 4.1 and Corollary 4.2).

Our second purpose is to discuss the relation between the local move and the invariants of $n$-knots. In the case of 1 -links, there is a great deal known about relations between local moves and knot invariants. (See [V,Wi,Ka2].) Our discussion is a high dimensional version of this theory.

\section{Definitions}

An (oriented) (ordered) $m$-component $n$-(dimensional) link is a smooth, oriented submanifold $L=\left\{K_{1}, \ldots, K_{m}\right\}$ of $S^{n+2}$, which is the ordered disjoint union of $m$ connected oriented submanifolds, each PL homeomorphic to the standard $n$-sphere. If $m=1$, then $L$ is called a knot. (This definition is used often. See $[\mathrm{CO}, \mathrm{L} 1, \mathrm{~L} 3]$.

Let $L_{1}$ and $L_{2}$ be $n$-links. $L_{1}$ is said to be equivalent to $L_{2}$ if there exists an orientation preserving diffeomorphism $h$ of $S^{n+2}$ such that $h \mid L_{1}$ is an orientation preserving diffeomorphism from $L_{1}$ to $L_{2}$. We work in the smooth category.

Definition. $\left(K_{1}, K_{2}\right)$ is called a pair of $n$-knots if $K_{1}$ and $K_{2}$ are $n$-knots. $\left(K_{1}, K_{2}, X_{1}, X_{2}\right)$ is called a 4 -tuple of $n$-knots and $(n+2)$-knots or a 4-tuple of $(n, n+2)$-knots if $\left(K_{1}, K_{2}\right)$ is a pair of $n$-knots and $X_{1}$ and $X_{2}$ are $(n+2)$-knots diffeomorphic to the standard $(n+2)$-sphere. $(n \geqq 1)$.

Received May 26, 1998.

This research was partially supported by Research Fellowships of the Promotion of Science for Young Scientists. 
Definition. A 4-tuple of $(n, n+2)$-knots $\left(K_{1}, K_{2}, X_{1}, X_{2}\right)$ is said to be realizable if there exists a smooth transverse immersion $f: S_{1}^{n+2} \amalg S_{2}^{n+2} \rightarrow S^{n+4}$ satisfying the following conditions: $(n \geqq 1)$

(1) The intersection $\Sigma=f\left(S_{1}^{n+2}\right) \cap f\left(S_{2}^{n+2}\right)$ is PL homeomorphic to the standard $n$-sphere.

(2) $f^{-1}(\Sigma)$ in $S_{i}^{n+2}$ defines an $n$-knot $K_{i}(i=1,2)$.

(3) $f \mid S_{i}^{n+2}$ is an embedding. $f\left(S_{i}^{n+2}\right)$ in $S^{n+4}$ is equivalent to $X_{i}(\mathrm{i}=1,2)$.

A pair of $n$-knots $\left(K_{1}, K_{2}\right)$ is said to be realizable or is called an intersectional pair of $n$-knots if there is a realizable 4-tuple of $(n, n+2)$-knots $\left(K_{1}, K_{2}, X_{1}, X_{2}\right)$.

\section{Intersectional pair of $n$-knots}

Our main theorem is:

Theorem 3.1. A pair of $n$-knots $\left(K_{1}, K_{2}\right)(n \geqq 1)$ is realizable if and only if $\left(K_{1}, K_{2}\right)$ satisfies the condition that

$$
\begin{cases}\left(K_{1}, K_{2}\right) \text { is arbitrary } & \text { if } n \text { is even, } \\ \operatorname{Arf}\left(K_{1}\right)=\operatorname{Arf}\left(K_{2}\right) & \text { if } n=4 m+1, \quad(m \geqq 0) . \\ \sigma\left(K_{1}\right)=\sigma\left(K_{2}\right) & \text { if } n=4 m+3,\end{cases}
$$

There is a mod 4 periodicity in dimension. It is similar to the periodicity in knot cobordism theory ([L1]) and surgery theory (see [Br,Wa,CS,We]). We have the following result on the realization of 4 -tuples of $(n, n+2)$-knots.

Theorem 3.2. A 4-tuple of $(n, n+2)$-knots $\left(K_{1}, K_{2}, X_{1}, X_{2}\right)$ is realizable if $K_{1}$ and $K_{2}$ are slice $(n \geqq 1)$. In particular, if $n$ is even, an arbitrary 4 -tuple of $(n, n+2)$-knots $\left(K_{1}, \bar{K}_{2}, X_{1}, X_{2}\right)$ is realizable.

Remarks. 1. Kervaire proved that all even dimensional knots are slice ([Ke]). 2. In [O1] the author discussed the case of two 3 -spheres in a 5-sphere. In [O2] the author discussed the case of the intersection of three 4-spheres.

Problem. Which 4 -tuples of $(2 n+1,2 n+3)$-knots are realizable $(n \geqq 1)$ ?

\section{High-dimensional pass-moves}

In order to prove Theorem 3.1, we introduce a new local move for high dimensional knots, the high dimensional pass-move. Pass-moves for 1-knots are discussed in p.146 of [Ka]. We define high dimensional pass-moves for $(2 k+1)$ knots $\subset S^{2 k+3},(k \geq 1)$.

Definition. Take a trivially embedded $(2 k+3)$-ball $B=B^{2 k+2} \times[-1,1]$ in $S^{2 k+3}$. We define $J_{+}, J_{-} \subset B$ as follows. (See Figure 4.1.) In $\partial B^{2 k+2} \times\{0\}$, take trivially embedded $S_{1}^{k}, S_{2}^{k}$ such that $\operatorname{lk}\left(S_{1}^{k}, S_{2}^{k}\right)=1$. Let $N\left(S_{*}^{k}\right)$ be a tubular neighborhood of $S_{*}^{k}$ in $\partial B^{2 k+2} \times\{0\}$. Let $h^{k+1}$ be a $(2 k+2)$-dimensional $(k+1)$ handle which is attached to $\partial B^{2 k+2} \times\{0\}$ along $N\left(S_{1}^{k}\right)$ with the trivial framing and which is embedded trivially in $B^{2 k+2} \times\{0\}$. Let $h_{+}^{k+1}$ (resp. $h_{-}^{k+1}$ ) be a $(2 k+2)$-dimensional $(k+1)$-handle which is embedded in $B=B^{2 k+2} \times[0,1]$ (resp. 
$\left.B=B^{2 k+2} \times[-1,0]\right)$ and which is attached to $\partial B^{2 k+2} \times\{0\}$ along $N\left(S_{2}^{k}\right)$ with the trivial framing. Let $h_{+}^{k+1} \cap h_{-}^{k+1}=N\left(S_{2}^{k}\right)$. Let $h_{+}^{k+1} \cap h^{k+1}=h_{-}^{k+1} \cap h^{k+1}=\phi$. Let $J_{+}$be the submanifold $\overline{\left(\partial h^{k+1}\right)-N\left(S_{1}^{k}\right)} \amalg \overline{\left(\partial h_{+}^{k+1}\right)-N\left(S_{2}^{k}\right)}$ in $B$. Let $J_{-}$ be the submanifold $\overline{\left(\partial h^{k+1}\right)-N\left(S_{1}^{k}\right)} \amalg \overline{\left(\partial h_{-}^{k+1}\right)-N\left(S_{2}^{k}\right)}$ in $B$. $\{0\}$.

In Figure 4.1 , we draw $B=B^{2 k+2} \times[-1,1]$ by using the projection to $B^{2 k+2} \times$

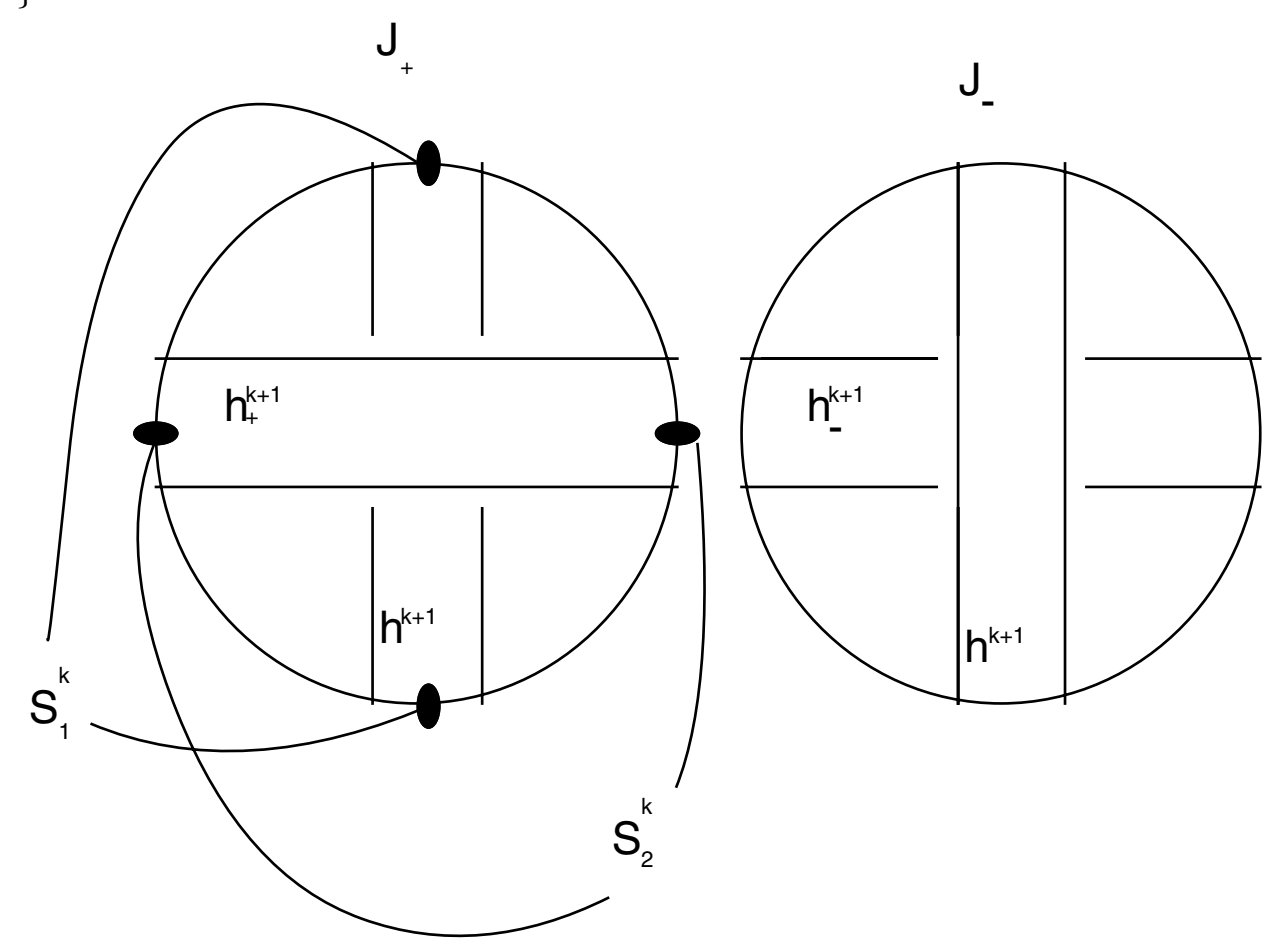

Figure 4.1

Let $K_{+}, K_{-}$be $(2 k+1)$-knots $\subset S^{2 k+3}$. We say that $K_{+}$is obtained from $K_{-}$ by one high dimensional pass-move if there is a trivially embedded $(2 k+3)$-ball $B \subset S^{2 k+3}$ such that $K_{+} \cap B$ is $J_{+}$and $K_{-} \cap B$ is $J_{-}$.

Let $K, K^{\prime}$ be $(2 k+1)$-knots $\subset S^{2 k+3}$. We say that $K$ is pass-move equivalent to $K^{\prime}$ if there are $(2 k+1)$-knots $K_{1}=K, K_{2}, \ldots, K_{\mu-1}, K_{\mu}=K^{\prime}(\mu \in \mathbb{N})$ such that $K_{i}$ is pass-move equivalent to $K_{i+1}$.

We prove the following:

Theorem 4.1. For $(2 k+1)$-knots $K_{1}$ and $K_{2}$, the following two conditions are equivalent: $(k \geqq 1)$

(1) There exists a $(2 k+1)$-knot $K_{3}$ which is pass-move equivalent to $K_{1}$ and cobordant to $K_{2}$.

(2) $K_{1}$ and $K_{2}$ satisfy the condition $\begin{cases}\operatorname{Arf}\left(K_{1}\right)=\operatorname{Arf}\left(K_{2}\right) & \text { when } k \text { is even, } \\ \sigma\left(K_{1}\right)=\sigma\left(K_{2}\right) & \text { when } k \text { is odd. }\end{cases}$

The $k=0$ case of Theorem 4.1 follows from [Ka]. 
Corollary 4.2. Let $K_{1}$ and $K_{2}$ be $(2 k+1)$-knots $(k \geqq 1)$. Suppose that $K_{1}$ is pass-move equivalent to $K_{2}$. Then $K_{1}$ and $K_{2}$ satisfy the condition that

$$
\begin{cases}\operatorname{Arf}\left(K_{1}\right)=\operatorname{Arf}\left(K_{2}\right) & \text { when } k \text { is even, } \\ \sigma\left(K_{1}\right)=\sigma\left(K_{2}\right) & \text { when } k \text { is odd. }\end{cases}
$$

Note. In [O3] the author proved a relation between another local move of 2 -knots and other invariants of 2-knots.

\section{Proof of Theorem 3.1}

We prove the following lemmas by explicit construction.

Lemma 5.1. Let $K$ be an $n$-knot. Then the pair of $n$-knots $(K, K)$ is realizable $(n \geqq 1)$.

Lemma 5.2. Let $K_{1}$ and $K_{2}$ be $(2 k+1)$-knots. Suppose that $K_{1}$ is pass-move equivalent to $K_{2}$. Then the pair of $(2 k+1)$-knots $\left(K_{1}, K_{2}\right)$ is realizable $(k \geqq 0)$.

Lemma 5.3. Let $K_{1}, K_{2}$ and $K_{3}$ be n-knots $(n \geqq 1)$. Suppose that the pair of $n$-knots $\left(K_{1}, K_{2}\right)$ is realizable and that $K_{2}$ is cobordant to $K_{3}$. Then the pair of $n$-knots $\left(K_{1}, K_{3}\right)$ is realizable.

Theorem 3.1 is deduced from Theorem 4.1 and Lemmas 5.1, 5.2, 5.3.

\section{Proof of Theorem 3.2}

It suffices to prove that a 4 -tuple of $(n, n+2)$-knots $\left(K_{1}, K_{2}, T, T\right)$ is realizable, where $K_{1}$ is a slice $n$-knot, $K_{2}$ is the trivial $n$-knot, $T$ is the trivial $(n+2)$-knot. Any 1-twist spun knot is unknotted ([Z]). Theorem 3.2 follows from this fact.

\section{The proof of Theorem 4.1}

Every $p$-knot $(p>1)$ is cobordant to a simple knot. (See [L1] for a proof and the definition of simple knots.) By using this fact, we prove that the $k \geqq 1$ case of Theorem 4.1 can be deduced from Theorem 7.1.

Proposition 7.1. For simple $(2 k+1)$-knots $K_{1}$ and $K_{2}$, the following two conditions are equivalent: $(k \geqq 1)$

(1) $K_{1}$ is pass-move equivalent to $K_{2}$.

(2) $K_{1}$ and $K_{2}$ satisfy the condition $\begin{cases}\operatorname{Arf}\left(K_{1}\right)=\operatorname{Arf}\left(K_{2}\right) & \text { when } k \text { is even, } \\ \sigma\left(K_{1}\right)=\sigma\left(K_{2}\right) & \text { when } k \text { is odd. }\end{cases}$

Proof of Proposition 7.1. (2) $\Rightarrow(1)$. $K_{1}$ bounds a Seifert hypersurface $V_{1}$ with a handle decomposition (one 0 -handle $) \cup((\mathrm{k}+1)$-handles). Take a Seifert matrix associated with $V_{1}$. By using high dimensional pass moves, we can change the Seifert matrix without changing the diffeomorphism type of $V_{1}$. Thus we obtain a $(2 k+1)$-knot $K_{2}^{\prime}$ whose Seifert matrix is same as the Seifert matrix of $K_{2}$ if (2) holds. By the classification theorem of simple knots by [L2], $K_{2}^{\prime}$ is equivalent to $K_{2}$. 
(1) $\Rightarrow(2)$. Suppose that $(2 k+1)$-knots $K_{*} \subset S_{*}^{2 k+3}$ bounds a Seifert hypersurface $V_{*}$. Note $V_{*}$ are $(2 k+2)$-manifolds. There is a compact oriented parallelizable $(2 k+4)$-manifold $P$ whose boundary is $S_{1}^{4 k+3} \amalg S_{2}^{4 k+3}$ containing compact oriented $(2 k+3)$-manifold $Q$ whose boundary is $V_{1} \cup\left(S^{2 k+1} \times[1,2]\right) \cup V_{2}$. (Here, $\partial V_{*}$ is $K_{*}$ and $S^{2 k+1} \times\{*\}$ is $K_{*}$.) We use characteristic classes and intersection products to prove $(1) \Rightarrow(2)$.

\section{Intersectional pair of submanifolds}

In $\S 1$ suppose $M$ is not PL homeomorphic to the standard sphere. Then we obtain a pair of submanifolds, $M$ in $S_{i}^{n+2}(i=1,2)$. Let $N$ be a closed oriented manifold. $\left(K_{1}, K_{2}\right)$ is called a pair of submanifolds (diffeomorphic to $N$ ) if $K_{i}$ is a submanifold of $S^{n+2}$ diffeomorphic to $N$.

Let $\left(K_{1}, K_{2}\right)$ be a pair of submanifolds diffeomorphic to $M$. We say $\left(K_{1}, K_{2}\right)$ is an intersectional pair if the submanifold $K_{i}$ is equivalent to the submanifold $M=S_{1}^{n+2} \cap S_{2}^{n+2}$ in $S_{i}^{n+2}$ as in $\S 1(i=1,2)$. It is natural to ask the following problem.

Problem 8.1. Which pairs of submanifolds are intersectional pairs?

The author can prove the following results. When $n$ is even, not all pair of submanifolds as above are realizable. When $n=4 m+3$, we can define the signature as in the knot case and the signature is an obstruction. Therefore not all pairs are realizable. When $n=3,\left(K_{1}, K_{2}\right)$ is realizable if and only if $\sigma\left(K_{1}\right)=\sigma\left(K_{2}\right)$. When $n \neq 3, \sigma\left(K_{1}\right)=\sigma\left(K_{2}\right)$ does not imply $\left(K_{1}, K_{2}\right)$ is realizable in general. When $n=4 m+1$, there is a closed oriented manifold $M$ such that if $K_{1}$ and $K_{2}$ are PL homeomorphic to $M$, then $\left(K_{1}, K_{2}\right)$ is realizable. In other words, there is no invariant corresponding to the Arf invariant as in the knot case. Of course, not all pairs are realizable.

\section{Acknowledgements}

The author would like to thank Prof. Levine for his interest in this paper, his invitation for the author, and for his help in correcting the author's English.

The author would also like to thank the referee and the editors for their reading of the manuscript with patience.

\section{References}

[Br] W. Browder, Surgery on simply-connected manifolds, Springer-Verlag, New York, 1972.

[CS] S. Cappell and J. Shaneson, The codimension two placement problem and homology equivalent manifolds, Ann. of Math. 99 (1974).

[CO] T. Cochran and K. Orr, Not all links are concordant to boundary links, Ann. of Math. 138 (1993), 519-554.

[Ka] L. Kauffman, Formal knot theory, Princeton Univ. Press Math. Notes 30 (1983).

[Ka2] Knots and Physics, World Scientific, Series on Knots and Everything 1 (1991).

[Ke] M. Kervaire, Les nœuds de dimensions supérieures, Bull. Soc. Math. France 93 (1965), $225-271$.

[L1] J. Levine, Knot cobordism in codimension two, Comment. Math. Helv. 44 (1969), 229244. 
[L2] An algebraic classification of some knots of codimension two, Comment. Math. Helv. 45 (1970), 185-198.

[L3] _ Link invariants via the eta-invariant, Comment. Math. Helv. 69 (1994), 82119.

[O1] E. Ogasa, The intersection of spheres in a sphere and a new geometric meaning of tt, University of Tokyo Preprint.

[O2] The intersection of three spheres in a sphere and a new application of the Sato-Levine invariant, Proc. Amer. Math. Soc. 126 (1998), 3109-3116.

[O3] Ribbon moves of 2-links: The $\mu$-invariants of 2-links and Tor $H_{1}(\mathbb{Z})$ of Seifert hypersurfaces, Preprint.

[V] V.A. Vassiliev, Complements of discriminants of smooth maps: topology and applications, Translations of Mathematical Monographs, Amer. Math. Soc. 98 (1992).

[Wa] C.T.C. Wall, Surgery on compact manifolds, London Mathematical Society Monographs, No. 1, Academic Press, London-New York, 1970.

[Wi] E. Witten, Quantum field theory and the Jones polynomial, Comm. Math. Phys. 121 (1989), 351-399.

[We] S. Weinberger, The topological classification of stratified spaces, Chicago Lectures in Mathematics, Univ. of Chicago Press, Chicago, Il, 1994.

Department of Mathematical Sciences, University of Tokyo, Komaba, Tokyo 153, JAPAN

Department of Mathematics, Brandeis University, Waltham, MA02254, USA

E-mail address: ogasa@ms.u-tokyo.ac.jp, ogasa@max.math.brandeis.edu 